\title{
Binocular detection by normal and stereoblind observers
}

\author{
DAVID H. WESTENDORF, ANDY LANGSTON, DEBBI CHAMBERS \\ and CHRISTINE ALLEGRETTI \\ University of Arkansas, Fayetteville, Arkansas 72701
}

\begin{abstract}
Binocular forced-choice detection performance was measured in three stereoblind observers and four observers with normal stereopsis. Detection rates of normal observers were greater than expected from probability summation, while those of the stereoblind observers were near or at a level expected from probability. It is concluded that binocular summation is reduced or absent in stereoblind persons.
\end{abstract}

In recent years, a number of studies of human binocular vision have focused on individuals who are unable to appreciate stereopsis. The results of these studies have supported the view that stereoblind individuals lack the binocular neurons of the visual cortex which may mediate stereopsis in the normal binocular visual system.

In particular, it has been shown that stereoblind humans frequently have a history of strabismus (Banks, Aslin, \& Letson, 1975; Hohman \& Creutzfeldt, 1975). Although there is no direct evidence concerning the physiological consequences of strabismus in humans, in other binocular animals, like cat, surgically induced strabismus imposed early in life results in a reduction in the proportion of cortical neurons which are binocularly innervated (Hubel \& Wiesel, 1965). It is relevant to point out that normally reared cats have stereopsis (Fox \& Blake, 1971), while cats raised without binocular experience do poorly in tasks requiring judgments of visual distance (Blake \& Hirsch, 1975).

The idea that stereoblind humans lack the normal complement of binocular cells is also supported by a number of recent studies demonstrating that these individuals do not show normal interocular transfer of aftereffects of motion (Mitchell, Reardon, \& Muir, 1975; Wade, 1976) and tilt (Mitchell \& Ware, 1974; Movshon, Chambers, \& Blakemore, 1972). The interocular transfer of visual aftereffects is generally interpreted as a reflection of binocular neural involvement. Thus, the reduced interocular transfer shown by stereoblind persons together with the link between stereoblindness and strabismus strongly suggests that these persons have a reduced number of binocular cells.

The purpose of the present experiment was to determine how stereoblind observers perform in a

Support for this research was provided in part by a grant from the University of Arkansas Research Reserve Fund and by an NIMH research grant (MH28063-01). binocular detection task. A number of studies have demonstrated that observers perform better with two eyes than with a single eye in threshold detection (e.g., Matin, 1962; Thorn \& Boynton, 1974; Westendorf, Blake, \& Fox, 1972) and a number of other visual tasks (for a recent review, see Blake \& Fox, 1973). This advantage of binocular viewing is referred to as binocular summation. In the case of threshold detection, binocular performance has been found to be superior to monocular by a margin greater than expected from probability summation and therefore must result from facilitatory neural interaction between the eyes. Since binocular summation, like stereopsis and interocular transfer, might logically result from binocularly innervated neurons, and since stereoblind observers are likely to have visual systems deficient in such neurons, we expected such observers would not exhibit the normally demonstrated advantage of using two eyes in the detection task.

\section{METHOD}

\section{Subjects}

Four normal and three stereoblind observers participated in the experiment. Two of the normal observers (D.C. and A.L.) were coauthors of this paper. The other two normal observers (G.M. and T.W.) were paid volunteers. All normal observers had normal or corrected-to-normal visual acuity, absence of any marked phoria, and good stereopsis.

To located stereoblind observers, a series of random element stereograms were shown via a polarized projection technique to a number of general psychology classes at the University of Arkansas. From a population of about 1,200 students who witnessed this "stereopsis demonstration," a total of 40 binocularly sighted but totally stereoblind individuals were identified. All but three of these stereoblind persons were judged unsuitable as observers in the present experiment for the following reasons. First, most of these individuals were strongly ambliopic; visual acuity was markedly impaired in one eye. Second, during haploscopic presentation of the background displays that were employed in the experiment (described below), most of these individuals were apparently unable to see both eyes' displays simultaneously. These displays contained some important monocular features (nonius markers) which were designed to aid observers in main- 
taining accurate eye alignment on the displays. Of the 40 stereoblind individuals we examined, only the three employed in the main experiment were able to see all of these monocular features simultaneously; the rest of the stereoblind persons apparently experienced wholesale interocular suppression when viewing these displays.

All three of the stereoblind observers who were paid to participate in the experiment had a history of strabismus during childhood. All three were in their early $20 \mathrm{~s}$ at the time of testing and were completely unable to see stereopsis with simple line stereograms, the Bausch and Lomb orthorater stereopsis test, or random element stereograms.

One stereoblind observer, R.V., had his eyes surgically realigned at age 2 . Orthorater tests revealed that he still had an esophoria of about 7 diopters and acuity in each eye of $20 / 25$ with contact lens correction. The second observer, D.G., has a slight esophoria of about 4 diopters and acuity in each eye of $20 / 25$ with optical correction. The third observer, D.H., has a slight exophoria of about 4 diopters and better than $20 / 20$ acuity in both eyes without correction. While both D.G. and D.H. were apparently diagnosed as strabismic during childhood, neither received any corrective treatment or orthoptic therapy.

Before participating in the experiment, each of the stereoblind observers was informally tested for binocular rivalry and interocular transfer of a motion aftereffect. To examine rivalry, each observer viewed a vertical square-wave grating with one eye and a horizontal grating with the other by means of a hand-held stereoscope. All three observers reported experiencing spontaneous rivalry alternations during a $1-\mathrm{min}$ inspection period. Only one observer, D.G., revealed a tendency for one eye to dominate unequally over the other. That observer estimated that the righteye image was dominant for about $60 \%$ to $70 \%$ of the viewing period.

To test for the interocular transfer of an aftereffect, each observer was first familiarized with the movement aftereffect by viewing binocularly a rotating spiral during a 1 -min adaptation period and a stationary spiral during a 30 -sec test period. Following familiarization trials, several interocular transfer trials were performed in which the rotating spiral was viewed by one eye and the stationary spiral by the other. Only one stereoblind observer, D.G., reported any transfer of the aftereffect, and then only when his right eye was used for adaptation and his left eye for viewing the test stimulus. D.G. maintained that the aftereffect in this case was "very faint" compared to that experienced binocularly or monocularly (same eye used for adaptation and test).

\section{Apparatus}

The major apparatus in the main experiment consisted of a haploscope with main arms $1 \mathrm{~m}$ long. Each main arm had an auxiliary arm attached at a right angle. The haploscope and related apparatus were fitted with baffles to prevent stray light interference. The viewing port was fitted with an adjustable bite bar, headrest, and trial frames. The trial frames carried $2-\mathrm{mm}$ artificial pupils and, if required, lenses ground to the observer's prescription.

Background displays were mounted toward the ends of the main arms of the haploscope $86 \mathrm{~cm}$ from the eyes of the observer and transilluminated by incandescent bulbs powered by a wellregulated dc power supply. Test stimuli were light flashes generated in lamp houses mounted on the auxiliary arms of the haploscope by fluorescent tubes ( $\mathrm{F} 6 \mathrm{~T} 5 / \mathrm{CW}$ ) powered and controlled by an Iconix tachistoscope. The flashes were at the same optical distance as the background displays and superimposed on those displays by beam splitters.

The basic background display configuration is illustrated in Figure 1. These displays were high-contrast photographic transparencies mounted on translucent Plexiglas sheets. Each eye's display consisted of a $3^{\circ} 20^{\prime} \times 7^{\circ}$ horizontally oriented white rectangular field which framed three $2^{\circ}$ squares arranged side by side. The central square provided a binocular fixation cross and

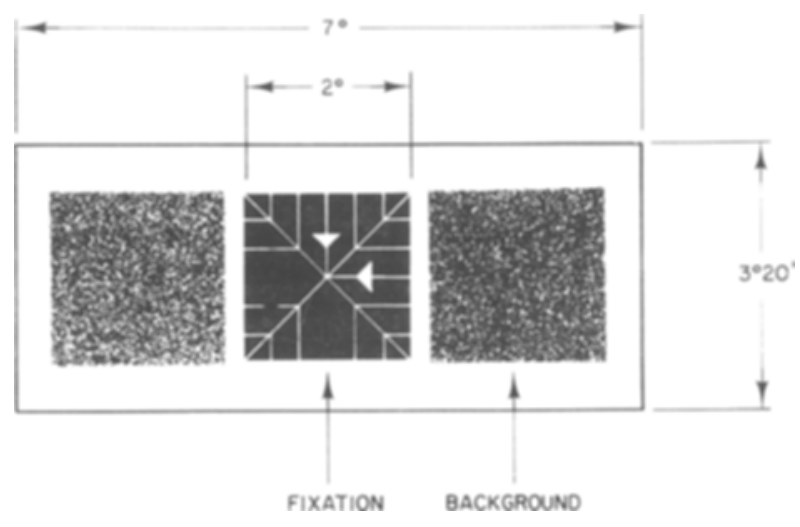

Figure 1. Background display. Test flashes were vertically oriented rectangular flashes superimposed near the center of the background square.

monocular nonius markers to facilitate accurate eye alignment. The flanking textured squares were each separated from the central square by $12^{\prime}$ and consisted of photographically reproduced shading material (Format 7149). Test flashes were superimposed at the center of the right-hand textured square. (The left-hand square was not functional in the present experiment.) The luminance of white portions of the background displays was set at $16 \mathrm{~cd} / \mathrm{m}^{2}$; textured-square space-averaged luminance was $8 \mathrm{~cd} / \mathrm{m}^{2}$; black portions were less than $.005 \mathrm{~cd} / \mathrm{m}^{2}$. Luminance was measured and periodically checked during experimental sessions with a Spectra brightness spotmeter (Model UB).

Test stimuli were $5^{\prime} \times 50^{\prime}$ vertically oriented rectangular flashes of $10 \mathrm{msec}$ duration. Test-flash luminance was adjusted for each subject to achieve desired levels of monocular detectability.

\section{Procedure}

Detection thresholds were measured with a four-choice temporal forced-choice psychophysical procedure with .25 a priori probability of a correct guess. Observers sat in a comfortable adjustable seat and viewed the stimulus display with their heads immobilized by a bite bar. Tones marking the temporal intervals during which a test flash could occur were delivered over a loudspeaker, superimposed on a continuous background of white noise. The observer initiated test trials by activating a solid-state timing/logic network that controlled the following events constituting a trial; following a .25-sec delay, a series of .75-sec tone bursts, separated by $.25 \mathrm{sec}$, were presented over the speaker. A test flash occurred randomly within one of those tone intervals; flash onset occurred $100 \mathrm{msec}$ after the onset of the tone burst. Observers reported in which interval the test flash most likely occurred by pressing one of four button switches. Feedback was provided following each trial.

Prior to the experiment, each observer participated in several sessions devoted to apparatus alignment, practice in the detection task, and determination of the monocular test stimulus luminance that yielded about $40 \%$ correct detection. The monocular test stimulus luminances used in the formal experiments are shown in Table 1.

\section{Table 1}

Monocular Test Stimulus Luminance Employed for Each Subject $\left(\mathrm{cd} / \mathrm{m}^{2}\right)$

\begin{tabular}{|c|c|c|c|c|c|c|c|}
\hline & \multicolumn{4}{|c|}{ Normal Observers } & \multicolumn{3}{|c|}{$\begin{array}{c}\text { Stereoblind } \\
\text { Observers }\end{array}$} \\
\hline & D.C. & A.L. & G.M. & T.W. & D.G. & D.H. & R.V. \\
\hline Left & 5.4 & 5.1 & 5.1 & 6.0 & 7.2 & 5.0 & 6.3 \\
\hline Right & 5.2 & 5.3 & 4.9 & 5.8 & 6.2 & 5.3 & 6.8 \\
\hline
\end{tabular}




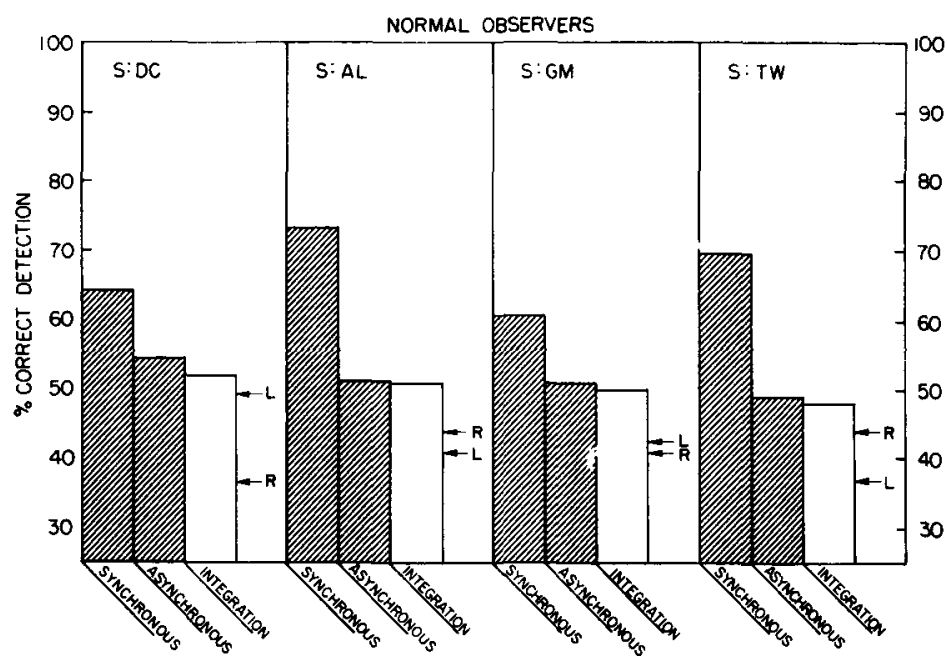

Figure 2. Results obtained from normal observers: Percentage of correct binocular detections (cross-hatched bars), integration model predictions of probability summation (white bars), and monocular detections (arrows).

In the formal experiment, detection thresholds were measured under four conditions. In one condition, binocular synchronous, a test stimulus was simultaneously presented to both eyes. In a second condition, binocular asynchronous, a test stimulus was presented to both eyes, but the flash to one eye was separated from that to the other by $100 \mathrm{msec}$. The binocular asynchronous condition was included to provide an empirical baseline for assessment of probability summation. In the third and fourth conditions, test stimuli were presented monocularly to the left and right eye, separately. Monocular thresholds were measured in order to compute expected probability summation performance employing a normative model of statistical independence.

From each observer, 500 judgements for each of these four conditions were obtained over 10 sessions. A session consisted of four randomized blocks of $\mathbf{5 0}$ trials each, with one block devoted to each condition. For the binocular asynchronous condition, which eye received the first test flash was counterbalanced across sessions. Under both monocular and binocular conditions, the observer kept both eyes open and maintained fusion of the background displays. To assure that stimulation was on corresponding retinal points, observers were frequently reminded to fixate the center of the fixation cross and never to initiate a trial if fusion was not present. Since, under prolonged inspection of the background displays, both normal and stereoblind observers occasionally experienced the phenomenal suppression of one or more of the monocular nonius markers, they were instructed to initiate trials only when all nonius markers were visible and binocularly aligned. If the observer reported that fusion was lost or suppression occurred during a trial, that trial was discarded and replaced. Prior to the first trial of a session, observers spent at least 10 min adapting to the stimulus field, during which time optical alignment of the system was checked, and at least one block of 50 practice trials was administered.

\section{RESULTS AND DISCUSSION}

The results from each of the four stereo-normal control observers are shown in Figure 2; those from each of the three stereoblind observers are illustrated in Figure 3. The two cross-hatched bars on the left of each panel show the percent-correct binocular detections of test stimuli presented synchronously and asynchronously. The white bar in each panel indicated predictions derived from a model of probability summation. That model, described below, yields predictions based on monocular performance, which is indicated by the arrows on the right side of each panel.

Binocular asynchronous condition performance is used as an empirical estimate of probability summation, that is, the level of performance expected if the two eyes only provide two chances to detect. The use of asynchronous conditions for obtaining empirical estimates is based on the work of Matin (1962), who found that test flashes separated by intervals greater than about $90 \mathrm{msec}$ do not interact except probabilistically. Wilcoxon

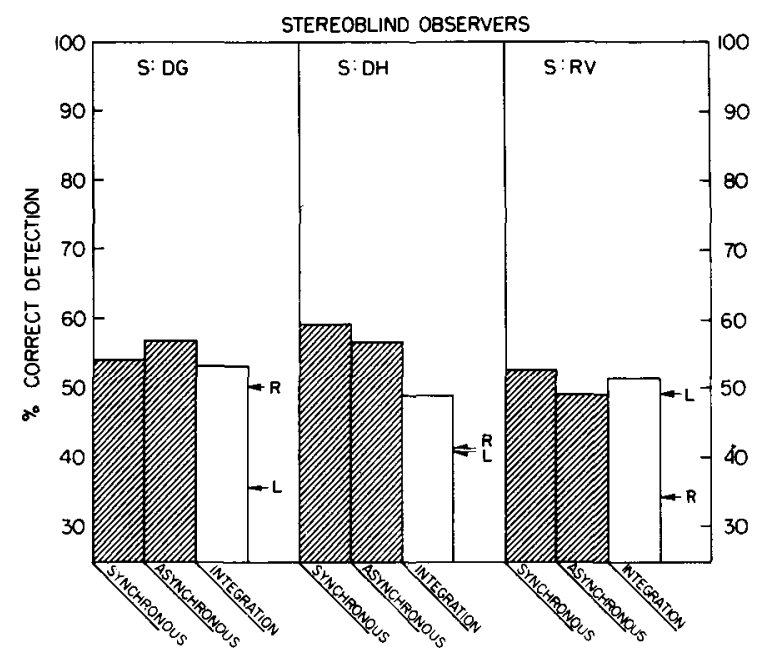

Figure 3. Results obtained from stereoblind observers: Percentage of correct binocular detections (cross-hatched bars), integration model predictions of probability summation (white bars), and monocular detections (arrows). 
signed-ranks tests on session-by-session detection rates were used to evaluate differences in detection performance under synchronous vs. asynchronous binocular stimulation. For each stereo-normal observer, these differences are significant (each $\mathrm{n}=10, \mathrm{p}<.01$ ); synchronous performance is superior to asynchronous, indicating summation in excess of probability for these observers. On the other hand, for all three stereoblind observers, differences between synchronous and asynchronous condition performance are small and in no case approach significance (each $\mathrm{n}=10, \mathrm{p}>.05$ ). Inspection of Figures 2 and 3 reveals that while stereo-normals have detection rates for synchronous flashes ranging from about $10 \%$ to $20 \%$ above their asynchronous hit rates, stereoblind observers have synchronous detection rates within $3 \%$ of their asynchronous performance. In fact, for two stereoblind observers, D.G. and R.V., binocular synchronous detection rates are not even significantly above their best-eye monocular rates (each $n=10$, $\mathrm{p}>$.05). The other stereoblind observer, D.H., showed a highly significant improvement in binocular synchronous detection relative to best-eye monocular performance $(n=10, p<.01)$.

While the safest approach to estimating probability summation is to use empirical estimates, an alternative approach is to use probability predictions computed from normative models of statistical independence. In the present experiment, we assessed monocular performance in order to compute expected probability from one such model of statistical independence, the integration model of Green and Swets (1966). The integration model is derived from signal detection theory and is particularly appropriate for estimating probability summation when detection rates involve a guessing component, as in the forced-choice method used in the present experiment. Several past experiments involving foveal presentation of test flashes found integration model predictions to agree rather closely with empirical estimates (Westendorf, Blake \& Fox, 1972; Westendorf \& Fox, 1974; 1975). But in one recent experiment (Westendorf \& Fox, 1977) which employed conditions of stimulation virtually identical to those of the present experiment, integration model predictions were found to be consistently below (by about $6 \%$ to $8 \%$ ) the performance with asynchronous stimulation. Our major purpose for computing integration model prediction in the present experiment was to determine whether this tendency toward underprediction noted by Westendorf and Fox (1977) was replicable.

The integration model predicts binocular sensitivity ( $\left.d^{\prime} b\right)$ by integrating the two monocular sensitivities ( $d{ }^{\prime} r$ and $\left.d^{\prime}{ }_{1}\right)$. Thus,

$$
d^{\prime} b=\sqrt{\left(d^{\prime} r\right)^{2}+\left(d^{\prime} 1\right)^{2}} \text {. }
$$

Monocular detection rates were converted to $\mathrm{d}^{\prime}$ values by using conversion tables prepared by Elliot (in Swets, 1964, appendix 1). An examination of Figure 2 shows that for all four stereo-normal observers integration model predictions are remarkably close to the detection rates obtained under the binocular asynchronous condition. The results for the stereoblind observers, shown in Figure 3, are less clear-cut. For Observers D.G. and R.V., the model predictions agree reasonably well with the empirical estimates of probability. However, for Observer D.H., the model prediction falls reliably below both asynchronous- and synchronouscondition performance levels. As assessed by the signed-ranks test on session-by-session performance, D.H.'s normative predictions are significantly below performance in the asynchronous and synchronous conditions (each $n=10, p<.01$ ). In spite of this result, overall, the integration model predictions are close to empirical estimates in the present experiment. Averaging across all seven subjects, the model predictions are only about $2 \%$ below asynchronous condition performance. The trend toward underprediction by the integration model, noted by Westendorf and Fox (1977), was replicated here at best very tenuously.

The difference between empirical and model estimates of probability for D.H. results in some uncertainty as to whether or not he showed binocular summation. Based on the empirical estimate, probability summation can account for his synchronous condition performance. But if the integration model estimate is used as the probability baseline, he showed summation. While empirical estimates are normally to be preferred, one could argue that in the present case the lower normative prediction constitutes a more conservative baseline for concluding that summation did not take place. We therefore believe it wise to leave open the possibility that some summation in excess of probability is reflected in D.H.'s synchronous condition performance. This conclusion is justified in light of the evidence from the present and several past experiments where integration model predictions were found generally close to empirical estimates. But based on the rather small difference between D.H.'s synchronous and asynchronous condition performance, it is reasonable to conclude that D.H. shows less summation than normal observers.

Our data show that summation in detection is reduced in stereoblind, relative to normal, observers. The most economical interpretation of the binocular detection performance of stereoblind observers is that it reflects only the binocular advantage expected from probability summation. This interpretation requires the reasonable assumption that the failure of observers D.G and R.V. to detect binocularity at a level significantly above their best-eye monocular rate is due to the large disparity in detection 
performance between the two eyes. Both D.G. and R.V. had detection rates in one eye not far above chance and well below the other eye's detection rates. Therefore, only a modest increment in detectability due to probability summation should be expected for these observers.

The conclusion that stereoblind observers show probability summation is of special interest in light of prior work on binocular summation with normal observers. Westendorf and Fox $(1974,1975)$ recently examined the binocular detection of stimulus combinations that induce binocular rivalry when viewed continuously above threshold. With such stimulus combinations as a positive light flash to one eye paired with a negative flash to the other (Westendorf \& Fox, 1974) or a vertical rectangular flash in one eye superimposed on a horizontal flash in the other (Westendorf \& Fox, 1975), detection performance was at a level expected from probability summation. Thus, although the stereoblind observers we tested might normally see with one eye at a time because of interocular suppression during suprathreshold viewing, both eyes can operate simultaneously, but independently, as detectors at the level of the forced-choice threshold. Furthermore, the present data suggest that the deficit in stereopsis need not be due to active interocular suppression per se but could result from the operation of the two eyes as independent detectors that fail to provide the eye-of-origin information requisite for stereopsis.

Our results both complement and serve as a counterpoint to those of several recent studies which found no difference between monocular and binocular performance by stereoblind observers in other visual tasks. Williams (1974) observed that in a dichoptic form identification task, requiring the binocular integration of monocular information over both time and space, stereoblind observers performed no better binocularly than monocularly while normal observers showed a binocular advantage in excess of predicted probability summation. Also, Lema and Blake (1977) reported that, compared to normal observers, stereoblind individuals show little or no improvement in binocular relative to monocular performance in a contrast detection task. While these results can be interpreted as due to interocular suppression, as proposed by Williams (1974), it is not clear to what extent they may also have been influenced by confounding factors like nonsynchronous eye movements or inaccurate binocular fixation. Furthermore, as Lema and Blake (1977) pointed out, methodological factors may have prevented the occurrence of probability summation in their experiment. The method of adjustment, which they used, might yield threshold contrasts which are near $100 \%$ detectable in a percent correct experiment. The results of the present experiment are relatively free of such problems of interpretation and can be taken to suggest that active inhibition between the eyes occurs relatively late in the sequence of events that characterize binocular information processing.

Finally, we should point out that the two stereoblind individuals examined by Williams (1974), three out of four of those tested by Lema and Blake (1977), and the three stereoblind observers in the present experiment had histories of strabismas. All of these individuals showed less than normal binocular summation in the various visual tasks they performed. These results are consistent with the view that stereoblindness is attributable to a reduction in the proportion of neurons that are binocularly innervated. Perhaps stereopsis, interocular transfer, and binocular summation are based on a common neural mechanism. That the same neurons mediate all these phenomena has not been demonstrated; but it has been shown that the neural mechanisms mediating each are similarly disrupted by abnormal early binocular experience.

\section{REFERENCES}

Banks, M. S., Aslin, R. N., \& Letsin, R. D. Critical period for the development of human binocular vision. Science, 1975, 190, 675-677.

BLAKE, R., \& Fox, R. The psychophysical inquiry into binocular summation. Perception \& Psychophysics, 1973, 14, 161-185.

Blake, R., \& HIRsch, H. V. B. Deficits in binocular depth perception in cats after alternating monocular deprivation. Science, 1975, 190, 1114-1116.

Fox, R., \& Blake, R. R. Stereoscopic vision in the cat. Nature. New Biology, London, 1971, 233, No. 5314, 55-56.

GREen, D. M., \& SwETs, J. A. Signal detection theory and psychophysics. New York: Wiley, 1966.

Hohman, A., \& Creutzfeldt, O. D. Squint and the development of binocularity in humans. Nature, London, 1975, 254, 613-614.

Hubel, D. H., \& Wiesel, T. N. Binocular interaction in striate cortex of kittens reared with artificial squint. Journal of Neurophysiology, 1965, 28, 1041-1059.

LEMA. S. A.. \& BLAKE, R. Binocular summation in normal and stereoblind humans. Vision Research, 1977, 17, 691-695.

Matin, L. Binocular summation at the absolute threshold for peripheral vision. Journal of the Optical Society of America, 1962. 52. 1276-1286.

Mitchell, D. E., Reardon, J., \& Muir, D. Interocular transfer of the motion aftereffect in normal and stereoblind observers. Experimental Brain Research, 1975, 22, 163-173.

Mitchell. D. E., \& WARE, C. Interocular transfer of a visual aftereffect in normal and stereoblind humans. Journal of Physiology, London, 1974, 236, 707-721.

Movshon, J. A., Chambers, B. E. I., \& Blakemore, C. Interocular transfer in normal humans, and those who lack stereopsis. Perception. 1972, 1, 483-490.

SWETS. J. A. Signal detection and recognition by human observers. New York: Wiley, 1964.

Thorn, F., \& Boynton, R. M. Human binocular summation at absolute threshold. Vision Research, 1974, 14, 445-458.

WADE. N.J. On interocular transfer of the movement aftereffect in individuals with and without normal binocular vision. Perception, 1976. 5. 113-118. 
Westendorf, D. H., Blake, R. R., \& Fox, R. Binocular summation of equal-energy flashes of unequal duration. Perception \& Psychophysics, 1972, 12, 445-448.

WestendorF, D. H., \& Fox, R. Binocular detection of positive and negative flashes. Perception \& Psychophysics, 1974, 15, 61-65.

Westendorf, D. H., \& Fox, R. Binocular detection of vertical and horizontal line segments. Vision Research, 1975, 15, 471-476.
WestendoRF, D. H., \& Fox, R. Binocular detection of disparate light flashes. Vision Research, 1977, 17, 697-702.

Williams, R. The effect of strabismus on dichoptic summation of form information. Vision Research, 1974, 14, 307-309.

(Received for publication February 9, 1978; revision accepted June 14, 1978.)

\section{ERRATUM}

Gregory, A. H. Perception of clicks in music. Perception \& Psychophysics, 1978, 24, 171-174-In the first line of the Discussion section, on page 173, the word "not" should be deleted. The line correctly should read: "Clicks do seem to be attracted towards phrase...." 